Pacific Journal of Mathematics

PSEUDO-COMPLETENESS AND THE PRODUCT OF BAIR

UN ARRIS AND DAViD JOHN LUTE 


\title{
PSEUDO-COMPLETENESS AND THE PRODUCT OF BAIRE SPACES
}

\author{
J. M. AARTS AND D. J. LUtZER
}

\begin{abstract}
The class of pseudo-complete spaces defined by Oxtoby is one of the largest known classes $\mathscr{C}$ with the property that any member of $\mathscr{C}$ is a Baire space and $\mathscr{C}$ is closed under arbitrary products. Furthermore, all of the classical examples of Baire spaces belong to $\mathscr{C}$. In this paper it is proved that if $X \in \mathscr{C}$ and if $Y$ is any (quasi-regular) Baire space, then $X \times Y$ is a Baire space. The proof is based on the notion of $A$-embedding which makes it possible to recognize whether a dense subspace of a Baire space is a Baire space in its relative topology. Finally, examples are presented which relate pseudo-completeness to several other types of completeness.
\end{abstract}

1. Introduction. A space $X$ is a Baire space if every nonempty open subset is of second category [2] or, equivalently, if the intersection of countably many dense open subsets of $X$ is dense in $X$. Locally compact Hausdorff spaces and completely metrizable spaces are the classical examples of Baire spaces.

In [10] Oxtoby introduced the notion of a pseudo-complete space (see $\S 2$ for precise definitions). Pseudo-complete spaces are Baire spaces and the classical examples of Baire spaces are pseudo-complete. Also, Cech-complete spaces (i.e., $G_{\delta}$-subsets of compact Hausdorff spaces [3]) as well as subcompact spaces [6] belong to the class of pseudocomplete spaces.

Pseudo-completeness has nice invariance properties. In particular, the topological product of any family of pseudo-complete spaces is pseudo-complete. Thus such a product is a Baire space.

In dealing with pseudo-completeness, assumptions about the usual separation axioms are irrelevant. However, it is often convenient to consider spaces which are quasi-regular, i.e., every nonempty open set contains the closure of some nonempty open set (cf. [10]).

Oxtoby [10] has also given an example of a completely regular Baire space whose square is not a Baire space, thus showing that a product theorem for Baire spaces cannot be obtained without some additional condition on (at least one of) the factors.

The main result of our paper is that the product of a quasi-regular Baire space and a pseudo-complete space is a Baire space. The techniques employed here, especially those in $\S 4$, are quite different from the usual category type techniques.

This paper is organized as follows. In $\S 2$ we discuss some new 
results on pseudo-completeness and its relation to some other completeness concepts. In $\S 3$ the notion of an $A$-embedded subset is introduced. It is shown that the property of being an $A$-embedded subset of a Baire space $Z$ is in a sense complementary to that of being a subset (of $Z$ ) which is a Baire space. Finally, in $\S 4$ the product theorem is proved.

We adopt the following notational convention: given a sequence $S_{1}, S_{2}, \cdots$ of subsets of a set $X$ we write $\bigcap\left\{S_{n}\right\}$ instead of $\bigcap\left\{S_{n} \mid n=\right.$ $1,2,3, \cdots\}$.

2. Pseudo-complete spaces. In this section all spaces are assumed to be quasi-regular (see $\S 1$ ).

2.1. We begin with a brief review of results about pseudocompleteness from [10].

Definitions. A pseudo-base for $X$ is a collection $\mathscr{P}$ of nonempty open sets such that each nonempty open $U \subset X$ contains some member of $\mathscr{T}$. A space $X$ is pseudo-complete if $X$ has a sequence $\{\mathscr{P}(n)\}$ of pseudo-bases such that if $P_{n} \in \mathscr{P}(n)$ and $\operatorname{cl} P_{n+1} \subset P_{n}$ for each $n$, then $\bigcap\left\{P_{n}\right\} \neq \phi$.

THEOREM. (a) Any dense $G_{\dot{\delta}}$-subset of a countably compact space is pseudo-complete.

(b) Any product of pseudo-complete spaces is pseudo-complete.

(c) Any pseudo-complete space is a Baire space.

In view of (a) the classical examples of Baire spaces as well as Cech-complete spaces are pseudo-complete. The pseudo-completeness of a subcompact space easily follows from the definitions (if $X$ is subcompact relative to the base $\mathscr{C}$, let $\mathscr{P}(n)=\mathscr{C}[6])$.

2.2. We now mention without proofs some simple facts about pseudo-completeness which are not in [10].

Proposition. (a) Let $X$ be a dense subspace of $Y$. If $X$ is pseudocomplete, then so is $Y$.

(b) Any topological sum (i.e., disjoint union) of pseudo-complete spaces is pseudo-complete.

(c) Any open subspace of a pseudo-complete space is pseudocomplete.

It is easily seen that closed subspaces of pseudo-complete spaces need not be pseudo-complete. For example, Michael's line-the set 
of real numbers topologized by taking sets of the form $U \cup V$ to be open, where $U$ is open in the usual topology of the reals and $V$ is any set of irrationals-is pseudo-complete (in view of (a)) and yet contains the usual space of rational numbers as a closed subspace.

Observe that the proposition above remains valid if "pseudo-complete space" is replaced by "Baire space". The following corollaries hold for any property of topological spaces for which the corresponding propositions (a), (b), and (c) hold.

CoROLlaRY 1. If a space $X$ has an open almost-cover (i.e., a collection of open sets whose union is dense in $X, c f$. [4]) by pseudocomplete spaces, then $X$ is pseudo-complete.

CoRollary 2. A space is locally pseudo-complete (i.e., each point has a neighborhood which is pseudo-complete) if and only if it is pseudo-complete.

2.3. The following result is related to a theorem of McCoy about Baire space extensions [9].

THEOREM. Any (quasi-regular) space $X$ is a dense subspace of a pseudo-complete space $\tilde{X}$.

Proof. The proof uses standard techniques; we present only an outline. The main difference between our construction and the standard ones is that we do not identify points of $X$ with (open) ultrafilters (since this would require separation axioms of $X$ ). Let $\mathscr{T}$ denote the topology of $X$. Let $\Delta$ denote the collection of all subfamilies of $\mathscr{T}$ which have the finite intersection property and are maximal with respect to this property. Let $\Omega=\left\{\xi \mid \xi \in \Delta\right.$ and $\left.\bigcap\left\{\mathrm{cl}_{X} F \mid F \in \xi\right\}=\phi\right\}$, the free elements of $\Delta$. Let $\widetilde{X}=X \cup \Omega$ (observe that the union is disjoint). For each $U \in \mathscr{T}$ let $\widetilde{U}=U \cup\{\xi \mid \xi \in \Omega$ and $U \in \xi\} . \quad \widetilde{X}$ is endowed with the topology for which $\{\tilde{U} \mid U \in \mathscr{T}\}=\tilde{\mathscr{T}}$ serves as a base. It is easily verified that any collection of open sets of $\tilde{X}$ having the finite intersection property has nonempty adherence in $\tilde{X}$. From $\operatorname{cl}_{\tilde{X}}(\tilde{U})=\tilde{U} \cup \operatorname{cl}_{X} U$ it follows that $\tilde{X}$ is quasi-regular (since $X$ is assumed to be quasi-regular).

By letting $\mathscr{P}(n)=\tilde{\mathscr{T}}$ for $n \geqq 1, \tilde{X}$ is shown to be pseudo-complete. In case $X$ is Hausdorff, $\widetilde{X}$ is a Hausdorff-closed extension of $X$ (cf. [7]).

2.4. As is well-known, the open continuous image of a Baire space is a Baire space. It is an open problem whether such mappings also preserve pseudo-completeness. However, if the range space is 
assumed to be metrizable, there is the following result.

Theorem. Suppose $X$ is pseudo-complete and $f: X \rightarrow Y$ is continuous, onto and open. If $Y$ is metrizable, then $Y$ contains a dense, completely metrizable, zero-dimensional subspace. In particular, $Y$ is pseudo-complete.

Proof. Let $\{\mathscr{P}(n)\}$ be a sequence of pseudo-bases for $X$ with respect to which $X$ is pseudo-complete. Inductively choose collections $\mathscr{P}^{\prime}(n) \subset \mathscr{P}(n)$ such that for each $n$ :

(a) If $P$ and $Q$ are distinct members of $\mathscr{P}^{\prime}(n)$, then $f(P) \neq f(Q)$;

(b) The collection $\mathscr{G}(n)=\left\{f(P) \mid P \in \mathscr{P}^{\prime}(n)\right\}$ is a disjoint open collection in $Y$ which is an almost cover ${ }^{1}$ of $Y$ with mesh $\leqq 1 / n$;

(c) $\mathscr{P}^{\prime}(n+1)$ refines $\mathscr{P}^{\prime}(n)$;

(d) If $P_{n+1} \in \mathscr{P}^{\prime}(n+1), P_{n} \in \mathscr{P}^{\prime}(n)$ and $f\left(P_{n+1}\right) \subset f\left(P_{n}\right)$, then $\mathrm{cl}_{X} P_{n+1} \subset P_{n}$.

Suppose $G_{n} \in \mathscr{G}(n)$ for $n \geqq 1$ and $G_{n} \supset G_{n+1}$. Because the collection $\mathscr{G}(n)$ is disjoint, it follows from (a) and (c) that there are unique members $P_{n}$ and $P_{n+1}$ of $\mathscr{P}^{\prime}(n)$ and $\mathscr{P}^{\prime}(n+1)$ respectively having $f\left(P_{n}\right)=G_{n}$ and $f\left(P_{n+1}\right)=G_{n+1}$. According to (d) we have $P_{n} \supset \operatorname{cl}_{X} P_{n+1}$. Therefore, $\bigcap\left\{P_{n}\right\} \neq \phi$. For any $x \in \bigcap\left\{P_{n}\right\}, f(x) \in \bigcap\left\{G_{n}\right\}$. In view of (b) $\{f(x)\}=\bigcap\left\{G_{n}\right\}$. For each $n$ let $W_{n}=\mathrm{U} \mathscr{G}(n)$, and let $Z=\bigcap\left\{W_{n}\right\}$. From the preceding observation it follows that $Z$ is a dense subset of $Y$. By virtue of (b), the collection $\mathscr{B}=\{G \cap Z \mid G \in \mathscr{G}(n), n=1$, $2, \cdots\}$ is a base for $Z$ consisting of relatively closed and open sets. The completeness of $Z$ is easily proved (cf. [1], [4], [6]).

CoRollary. A metrizable space $Y$ is pseudo-complete if and only if $Y$ contains a dense completely metrizable subspace (which may be taken to be zero-dimensional).

Proof. The "only if" part follows from the preceding theorem by taking $X=Y$. The "if" part follows from 2.1 Theorem (a) and 2.2 Proposition (a).

REMARK. F. G. Slaughter, Jr. pointed out to the authors that in the proofs of the theorem and corollary above it suffices to assume that $Y$ is quasi-regular and developable (instead of metrizable). Thus we have: a pseudo-complete Moore space contains a dense completely metrizable subspace.

The corollary above may be applied to show that pseudo-completeness and the property of being a Baire space are not equivalent, even

1 Cf. 2.2 Corollary 1. 
for metrizable spaces.

EXAMPLE. A space is said to be totally imperfect if it contains no uncountable compact subsets. According to a theorem of Bernstein, any separable, metrizable, complete and dense-in-itself space $X$ can be decomposed into two mutually disjoint totally imperfect subsets $Y$ and $Z$. Both $Y$ and $Z$ are known to be Baire spaces, but neither $Y$ nor $Z$ contains a dense completely metrizable subspace. See 2.6, 3.3 Example, and [8] for more details.

2.5. Problem. It is an open problem whether dense $G_{\hat{o}}$-subsets of pseudo-complete spaces are pseudo-complete. Observe that if dense $G_{i}$-subsets of pseudo-complete spaces and open continuous images of pseudo-complete spaces (see 2.4) are pseudo-complete, a less complicated proof of the Product Theorem 4.2 can be given.

2.6. A part of the theory we are going to present in $\S \S 3$ and 4 can be developed using other notions of "completeness" instead of pseudo-completeness. For example, we can define a completely regular space $X$ to be almost $\breve{C} e c h$-complete if $X$ contains a dense Čech-complete subspace (cf. [5]). The results of $\S 2.2$ also hold for almost Cechcomplete spaces and in view of 2.1 Theorem (a) such spaces are pseudo-complete, whence Baire spaces.

We next present an example which shows that the notion of pseudo-completeness is much more general than that of being almost Čech-complete.

ExAMPLE. There is a completely regular, pseudo-complete space $X$ such that each Čech-complete subspace $C$ of $X$ is nowhere dense (i.e., $\operatorname{int}_{X} \mathrm{cl}_{X} C=\phi$ ).

Let $Z=R^{c}=\Pi\left\{R_{r} \mid \gamma \in \Gamma\right\}$, the continuous product of real lines. Any nonempty $G_{o}$-subset of $Z$ has $\exp c$ points ${ }^{2}$ and there are exp $c$ nonempty $G_{\dot{o}}$-subsets of $Z$. We shall show that there is a subset $X$ of $Z$ such that neither $X$ nor $Z \backslash X$ contains any nonempty $G_{\dot{o}}$-subset of $Z$ and that $X$ has the above mentioned properties.

The construction of the set $X$ is very similar to that of a totally imperfect subset of a separable, metrizable, and dense-in-itself space as given in [8]. Well-order the collection of all nonempty $G_{\dot{o}}$-subsets of $Z$ as $\left\{H_{\alpha} \mid \alpha<\eta\right\}$ where $\eta$ is the first ordinal having cardinality $\exp c$. Using transfinite induction pick two distinct points $x_{\alpha}$ and $y_{\alpha}$ from each $H_{\alpha}$, taking care that at each step only points are picked

${ }^{2}$ We write $\exp c$ for $2^{c}$. 
which have not been selected before. $X$ is the set of all points $x_{\alpha}$ so obtained.

Now let $\mathscr{B}$ denote the collection of the basic open sets $\bigcap\left\{\pi_{\gamma}^{-1}\left(U_{\gamma}\right) \mid \gamma \in\right.$ $\Gamma_{0}$ \} where $\Gamma_{0} \subset \Gamma$ is finite and each $U_{r}$ is a bounded open interval in $R_{r}$. Let $\mathscr{P}(n)=\{B \cap X \mid B \in \mathscr{B}\}$ for each $n \geqq 1$. Observe that for each $B \in \mathscr{B}, \mathrm{cl}_{X}(B \cap X)=X \cap \mathrm{cl}_{Z} B$, because $X$ is dense in $Z$.

In order to prove that $X$ is pseudo-complete, suppose $P_{n} \in \mathscr{B}$ (so $\left.P_{n} \cap X \in \mathscr{P}(n)\right)$ and $\operatorname{cl}_{X}\left(P_{n+1} \cap X\right) \subset P_{n} \cap X$. Then

$$
\bigcap\left\{X \cap P_{n}\right\}=\bigcap\left\{\mathrm{cl}_{X}\left(X \cap P_{n}\right)\right\}=X \cap\left(\cap\left\{\mathrm{cl}_{Z} P_{n}\right\}\right) .
$$

Now, $\bigcap\left\{\mathrm{cl}_{z} P_{n}\right\}$ is nonempty because of the boundedness condition imposed on the basic open sets. Moreover, this intersection is a $G_{j^{-}}$ subset of $Z$. Hence its intersection with $X$ is nonempty. Thus $X$ is pseudo-complete.

If $D$ is a Čech-complete space which is dense in some open set $U$ of $X$, then $D$ is also dense in some open set $V$ of $Z$. Then $D \cap$ $V$ is a $G_{\dot{\delta}}$-subset of $Z$, so $(D \cap V) \cap(Z \backslash X) \neq \phi$. Hence $D$ is not contained in $X$.

3. $A$-embedded subsets. We shall now define the concept of an $A$-embedded subset, which plays a vital role in the proof of the Product Theorem.

3.1. Definition. Let $X$ be a subset of a space $Z$. Then $X$ is said to be $A$-embedded in $Z$ if each $G_{i}$-subset $H$ of $Z$ which is contained in $X$ is nowhere dense in $X$ (i.e., int $_{X} \operatorname{cl}_{X} H=\phi$ ).

EXAMPLE 1. The set $Q$ of the rational numbers is $A$-embedded in the real line $R$.

To prove this we first observe that in a countable $T_{1}$-space which is a Baire space, each open set has isolated points (since the complement of a non-isolated point is a dense open set). Now, let $H$ be a $G_{i}$-subset of $R$. If $H \subset Q$, then $H$ is countable, so that $H$ cannot be dense in any interval. It follows that $H$ is nowhere dense in $Q$.

ExAMPle 2. Let the subspace $S$ of $R$ be defined by $S=\{0\} \cup$ $\{1 / n \mid n=1,2, \cdots\}$. The set $\{0\}$ is not $A$-embedded in $S$.

Using the concept of $A$-embedded subsets, we are able to recognize subsets of Baire spaces which, in their relative topology, are Baire spaces.

3.2. THEOREM. Let $X$ be a dense subspace of a Baire space $Z$. (a) If $Z \backslash X$ is A-embedded in $Z$, then $X$ is a Baire space. 
(b) If $Z \backslash X$ is dense in $Z$, then $X$ is a Baire space if and only if $Z \backslash X$ is $A$-embedded in $Z$.

Proof. To prove (a), observe that if $X$ is not a Baire space, then there is a sequence $H \supset G_{1} \supset G_{2} \supset \cdots$ of open subsets of $X$ such that each $G_{n}$ is dense in $H$ and yet $\bigcap\left\{G_{n}\right\}=\phi$. Then there is a sequence $U \supset V_{1} \supset V_{2} \supset \cdots$ of open subsets of $Z$ such that $H=U \cap X$ and $G_{n}=V_{n} \cap X$. Each $V_{n}$ is dense in $U$ and $U$ is a Baire space. Hence $D=\bigcap\left\{V_{n}\right\}$ is dense in $U$ and therefore in $U \cap(Z \backslash X)$. Since $D \subset Z \backslash X$, $Z \backslash X$ is not $A$-embedded in $Z$.

To prove the "only if" part of (b), we assume that $Z \backslash X$ is not $A$-embedded in $Z$. Let $H$ be a $G_{\delta}$-subset of $Z$ which is contained in $Z \backslash X$ and which is dense in some relatively open set $U$ of $Z \backslash X$. Let $V$ be an open subset of $Z$ with $V \cap(Z \backslash X)=U$.

Then $F=V \cap H$ is a $G_{i}$-subset of $Z$ which is dense in $V$ and which is contained in $U$. Let $F=\bigcap\left\{F_{n}\right\}$ where each $F_{n}$ is open in $Z$ and $F_{n} \subset V$. The sets $F_{n} \cap X$ are open and dense subsets of $V \cap X$ and yet $\cap\left\{F_{n} \cap X\right\}=\phi$. It follows that $V \cap X$ is not a Baire space. Consequently, $X$ is not a Baire space.

REMARK. As is clear from 3.1 Example 2, the hypothesis that $Z \backslash X$ is dense in $Z$ cannot be omitted from part (b) above.

3.3. In connection with Theorem 3.2 we have the following propositions which will be used in the next section.

LEMMA 1. In every quasi-regular space $X$ there are open subspaces $X_{P}$ and $X_{A}$ such that

(a) $X_{P}$ and $X_{A}$ are disjoint and $X_{P} \cup X_{A}$ is dense in $X$;

(b) $X_{P}$ is pseudo-complete;

(c) any pseudo-complete subspace of $X_{A}$ is nowhere dense in $X_{A}$.

Proof. Let $X_{P}$ be the union of all open subsets of $X$ which are pseudo-complete in their relative topology.

Let $X_{A}=X \mid c 1_{X} X_{P}$. The lemma follows from 2.2 Proposition and 2.2 Corollary 1. (Observe that $X_{P}$ and $X_{A}$ may be empty.)

Lemma 2. Let $X, X_{P}$ and $X_{A}$ be as in Lemma 1. Then $X$ is a Baire space if and only if $X_{A}$ is a Baire space.

Proof. Obvious.

Proposition. Let $X$ be a quasi-regular space such that any pseudocomplete subspace of $X$ is nowhere dense in $X$. The following properties 
are equivalent:

(a) $X$ is a Baire space;

(b) For every pseudo-complete space $Y$ such that $X$ is dense in $Y$, the subset $Y \backslash X$ is A-embedded in $Y$;

(c) For some pseudo-complete space $Y$ such that $X$ is dense in $Y$, the set $Y \backslash X$ is A-embedded in $Y$.

Proof. For any pseudo-complete space $Y$ such that $X$ is dense in $Y$, the set $Y \backslash X$ is dense in $Y$, because $X$ contains no pseudo-complete open subspaces. That (a) implies (b) now follows from 3.2 Theorem (b). Obviously (c) follows from (b). Finally, 3.2 Theorem (a) shows that (a) follows from (c).

EXAMPLE. According to the above proposition, the totally imperfect subspaces $Y$ and $Z$ of a separable, complete, dense-in-itself metric space $X$ in 2.4 Example are Baire spaces. The proposition can also be applied to spaces which are not totally imperfect. In the special case where $X$ is the Euclidean plane, construct $Y$ and $Z$ as in 2.4 Example and let $Y^{\prime}=Y \cup L$ where $L$ is a straight line in $X$. Then $Y^{\prime}$ and $X \backslash Y^{\prime}$ are each $A$-embedded subspaces of $X$ (so that each is a Baire space) even though $Y^{\prime}$ is not totally imperfect.

REMARK. It follows directly from Lemma 2 above that a $\sigma$-locally compact space $X$ is a Baire space if and only if $X$ is pseudo-complete: one shows that the $\sigma$-locally compact Baire space $X_{A}$ must be empty.

\section{The product theorem.}

4.1. Projection Lemma. Suppose $X$ is a Baire space and $Y$ is pseudo-complete. If $D$ is a dense $G_{j}$-subset of $X \times Y$, then $\pi_{X}(D)$ contains a dense $G_{\delta}$-subset of $X$ (where $\pi_{X}$ denotes the natural projection of $X \times Y$ onto $X)$.

Proof. Write $D=\bigcap\left\{G_{n}\right\}$ where $G_{1} \supset G_{2} \supset \ldots$ are open subsets of $X \times Y$. Let $\{\mathscr{P}(n)\}$ be a sequence of pseudo-bases for $Y$ with respect to which $Y$ is pseudo-complete.

Let $\mathscr{G}(1)=\left\{U \mid \phi \neq U\right.$ is open in $X$ and $U \times P \subset G_{1}$ for some $P \in$ $\mathscr{P}(1)\}$. Let $\mathscr{V}(1)$ be a maximal disjoint subcollection of $\mathscr{U}(1)$. Since $D$ is dense in $X \times Y$, the set $W_{1}=\bigcup \mathscr{V}(1)$ is dense in $X$. For each $V \in \mathscr{O}(1)$ choose $P(V, 1) \in \mathscr{P}(1)$ such that $V \times P(V, 1) \subset G_{1}$.

Inductively define collections $\mathscr{V}(n)$ such that

(a) each $\mathscr{V}(n)$ is a disjoint collection of nonempty open subsets of $X$ and $W_{n}=\mathrm{U} \mathscr{V}(n)$ is dense in $X$;

(b) $\mathscr{V}(n+1)$ refines $\mathscr{V}(n)$; 
(c) for each $V \in \mathscr{V}(n)$ there is a member $P(V, n) \in \mathscr{P}(n)$ having $V \times P(V, n) \subset G_{n}$;

(d) if $V_{n+1} \in \mathscr{C}(n+1)$ and if $V_{n}$ is the unique member of $\mathscr{P}(n)$ containing $V_{n+1}$, then $\operatorname{cl}_{Y}\left(P\left(V_{n+1}, n+1\right)\right) \subset P\left(V_{n}, n\right)$.

Let $E=\bigcap\left\{W_{n}\right\}$. Since $X$ is a Baire space, $E$ is dense in $X$. To show that $E \subset \pi_{X}(D)$, let $x \in E$. Then there is a (unique) sequence $\left\{V_{n}\right\}$ such that $V_{n} \in \mathscr{V}(n)$ and $x \in V_{n}$. Necessarily $V_{n+1} \subset V_{n}$ so that $\operatorname{cl}_{Y}\left(P\left(V_{n+1}, n+1\right)\right) \subset P\left(V_{n}, n\right)$. Therefore, $\bigcap\left\{P\left(V_{n}, n\right)\right\} \neq \phi$ and for any $y \in \bigcap\left\{P\left(V_{n}, n\right)\right\},(x, y) \in G_{n}$ for each $n$ so that $(x, y) \in D$. Hence $x \in$ $\pi_{X}(D)$.

4.2. The Product Theorem. If $X$ is a quasi-regular Baire space and $Y$ is pseudo-complete, then $X \times Y$ is a Baire space.

Proof. As in 3.3 Lemma 1, choose open subsets $X_{P}$ and $X_{A}$ of $X$. Then $\left(X_{P} \times Y\right) \cup\left(X_{A} \times Y\right)$ is a dense subspace of $X \times Y$. Since $Y_{P} \times Y$ and $X_{A} \times Y$ are open subsets of $X \times Y$ and since $X_{P} \times Y$ is a Baire space in view of 2.1 Theorem (b) and (c), it is enough to show that $X_{A} \times Y$ is a Baire space. By 3.3 Lemma $2, X_{A}$ is a (quasi-regular) Baire space. Thus we need only consider the special case where $X=$ $X_{A}$.

In view of 2.3 Theorem, there is a pseudo-complete quasi-regular space $\tilde{X}$ which contains $X$ as a dense subspace. Let $X^{\prime}=\widetilde{X} \mid X$. Then $X^{\prime}$ is $A$-embedded in $\tilde{X}$ by virtue of 3.3 Proposition. Since $X \times$ $Y$ is a dense subset of the pseudo-complete space $\tilde{X} \times Y$, it will be sufficient to show that $X^{\prime} \times Y=(\tilde{X} \times Y) \backslash(X \times Y)$ is an $A$-embedded subset of $\tilde{X} \times Y$ (by virtue of 3.2 Theorem).

To this end, suppose $D \subset X^{\prime} \times Y$ is a $G_{j}$-subset of $\tilde{X} \times Y$ which is dense in some relatively open subset of $X^{\prime} \times Y$. Then there is a relatively open subset $G$ of $X^{\prime}$ and an open subset $V$ of $Y$ such that $D \cap(G \times V)$ is dense in $G \times V$. The set $G$ may be written as $G=$ $U \cap X^{\prime}$ where $U$ is open in $\widetilde{X}$. Then $D \cap(U \times V)$ is a dense $G_{i}$-subset of $U \times V$, the product of the Baire space $U$ with the pseudo-complete space $V$. According to the projection lemma, $\pi_{U}(D \cap(U \times V))$ contains a $G_{i}$-subset $E$ of $U$ which is dense in $U$. Then $E$ is a $G_{i}$-subset of $\widetilde{X}$. Furthermore, $E \subset \pi_{\widetilde{X}}(D) \subset X^{\prime}$ which is impossible because $X^{\prime}$ is known to be $A$-embedded in $\tilde{X}$.

It follows that $X^{\prime} \times Y$ is $A$-embedded in $\tilde{X} \times Y$ so that $X \times Y$ is a Baire space.

CoRollary. If $X$ is a quasi-regular Baire space and if the space $Y$ is (locally) compact Hausdorff or (locally) Čech-complete or (locally) subcompact, then $X \times Y$ is a Baire space. 
REMARK. In the Product Theorem the pseudo-completeness of $Y$ is by no means a necessary condition for $X \times Y$ to be a Baire space. Oxtoby [10] has proved that if $Y$ is any Baire space having a (locally) countable pseudo-base, then $X \times Y$ is a Baire space for any Baire space $X$. This result of Oxtoby and our product theorem overlap, but neither includes the other: (Cf. the examples in 2.4 and 2.6 of which the latter has no (locally) countable pseudo-base. Indeed there are compact Hausdorff spaces having no locally countable pseudo-base.) Furthermore, as has already been mentioned in the Introduction, the techniques employed in the proofs are totally different.

\section{REFERENCES}

1. J. M. Aarts, J. de Groot, and R. H. McDowell, Cotopology for metrizable spaces, Duke Math. J., 37 (1970), 291-295.

2. N. Bourbaki, Elements of Mathematics: General Topology, part 2, Addison-Wesley, Reading, Mass., 1966.

3. E. Čech, On bicompact spaces, Ann. of Math., 38 (1937), 823-844.

4. Z. Frolík, Generalizations of the $G_{\delta}$-property of complete metric spaces, Czech Math. J., 10 (1960), 359-379.

5. - Locally topologically complete spaces, Sov. Math. Dokl., 2 (1961), 355-357.

6. J. de Groot, Subcompactness and the Baire category theorem, Indag. Math., 25 (1963), 761-767.

7. M. Katětov, Über H-abgeschlossene und bikompakte Räume, Časopis Pěst. Mat. Fys., 69 (1940), 36-49.

8. C. Kuratowski, Topology, Vol. 1, Academic Press, New York, 1966.

9. R. McCoy, A Baire space extension, Proc. Amer. Math. Soc., 33 (1972), 199-202.

10. J. C. Oxtoby, Cartesian products of Baire spaces, Fund. Math., 49 (1961), 157-166.

Received June 30, 1972 and in revised form August 14, 1972. Partially supported by NSF Grant GP-29401.

Delft Institute of Technology, Delft, Netherlands

AND

UNIVERSITY OF PItTSBURgh 


\section{PACIFIC JOURNAL OF MATHEMATICS}

\section{EDITORS}

RICHARD ARENS (Managing Editor)

University of California

Los Angeles, California 90024

R. A. Beaumont

University of Washington

Seattle, Washington 98105
J. DUGUNDJI*

Department of Mathematics

University of Southern California

Los Angeles, California 90007

D. Gilbarg and J. Milgram

Stanford University

Stanford, California 94305

\section{ASSOCIATE EDITORS}
E. F. BECKENBACH
B. H. NeumanN
F. WOLF
K. YosHIDA

\section{SUPPORTING INSTITUTIONS}

UNIVERSITY OF BRITISH COLUMBIA
CALIFORNIA INSTITUTE OF TECHNOLOGY
UNIVERSITY OF CALIFORNIA
MONTANA STATE UNIVERSITY
UNIVERSITY OF NEVADA
NEW MEXICO STATE UNIVERSITY
OREGON STATE UNIVERSITY
UNIVERSITY OF OREGON
OSAKA UNIVERSITY

UNIVERSITY OF BRITISH COLUMBIA

UNIVERSITY OF CALIFORNIA

MONTANA STATE UNIVERSITY

UNIVERSITY OF NEVADA

OREGON STATE UNIVERSITY

OSAKA UNIVERSITY
UNIVERSITY OF SOUTHERN CALIFORNIA

STANFORD UNIVERSITY

UNIVERSITY OF TOKYO

UNIVERSITY OF UTAH

WASHINGTON STATE UNIVERSITY

UNIVERSITY OF WASHINGTON

\section{AMERICAN MATHEMATICAL SOCIETY} NAVAL WEAPONS CENTER

* C. R. DePrima California Institute of Technology, Pasadena, CA 91109, will replace J. Dugundji until August 1974. 


\section{Pacific Journal of Mathematics}

\section{Vol. 48, No. $1 \quad$ March, 1973}

Jan Aarts and David John Lutzer, Pseudo-completeness and the product of Baire

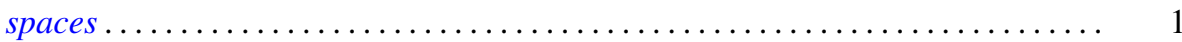

Gordon Owen Berg, Metric characterizations of Euclidean spaces ............ 11

Ajit Kaur Chilana, The space of bounded sequences with the mixed topology ..... . 29

Philip Throop Church and James Timourian, Differentiable open maps of

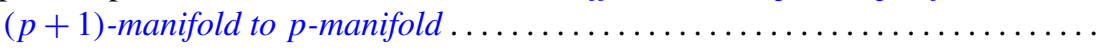

P. D. T. A. Elliott, On additive functions whose limiting distributions possess a finite

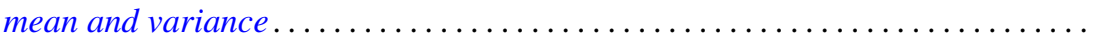

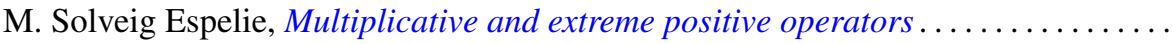

Jacques A. Ferland, Domains of negativity and application to generalized convexity

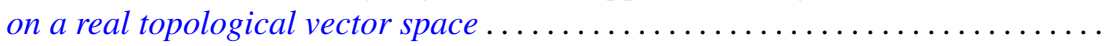

Michael Benton Freeman and Reese Harvey, A compact set that is locally holomorphically convex but not holomorphically convex ...............

Roe William Goodman, Positive-definite distributions and intertwining

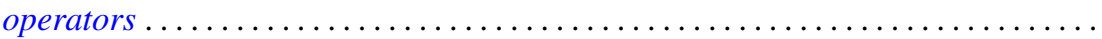

Elliot Charles Gootman, The type of some $C^{*}$ and $W^{*}$-algebras associated with

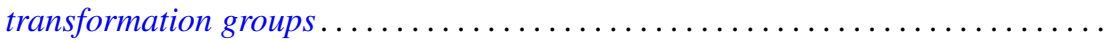

David Charles Haddad, Angular limits of locally finitely valent holomorphic

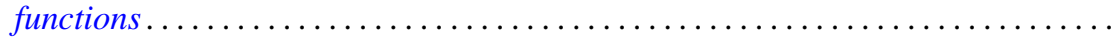

William Buhmann Johnson, On quasi-complements .

William M. Kantor, On 2-transitive collineation groups of finite projective spaces...

Joachim Lambek and Gerhard O. Michler, Completions and classical localizations of right Noetherian rings

Kenneth Lamar Lange, Borel sets of probability measures ......

David Lowell Lovelady, Product integrals for an ordinary differential equation in a Banach space

Jorge Martinez, A hom-functor for lattice-ordered groups .........

W. K. Mason, Weakly almost periodic homeomorphisms of the two sphere ....

Anthony G. Mucci, Limits for martingale-like sequences .......

Eugene Michael Norris, Relationally induced semigroups ...

Arthur E. Olson, A comparison of c-density and $k$-density ......

Donald Steven Passman, On the semisimplicity of group rings of linear groups.

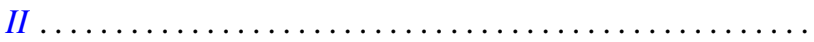

Charles Radin, Ergodicity in von Neumann algebras .

P. Rosenthal, On the singularities of the function generated by the Bergman operator of the second kind.

Arthur Argyle Sagle and J. R. Schumi, Multiplications on homogeneous spaces,

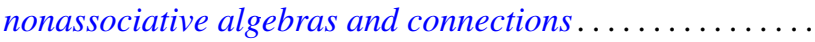

Leo Sario and Cecilia Wang, Existence of Dirichlet finite biharmonic functions on

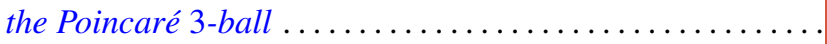

Ramachandran Subramanian, On a generalization of martingales due to Blake ..

Bui An Ton, On strongly nonlinear elliptic variational inequalities.

Seth Warner, A topological characterization of complete, discretely valued

fields. 